\title{
Three-dimensional ultrasound radiance mode imaging of a uterine lipoleiomyoma.
}

\author{
Francisco Raga ${ }^{1,2}$, Sara Cholvi ${ }^{1}$, Maria José Esquembre', Diana Boigues ${ }^{2}$, Carolina Lucas ${ }^{1}$, \\ Fernando Bonilla-Musoles ${ }^{2}$
}

${ }^{1}$ Servicio de Obstetricia y Ginecología, Hospital Clínico Universitario, ${ }^{2}$ Departamento de Obstetricia y Ginecología, Universidad de Valencia, Valencia, Spain

\begin{abstract}
In this case report the diagnosis of a uterine lipoleiomyoma is depicted by means of a three-dimensional radiance mode. The advent of radiance or silhouette mode as a new tool in ultrasound diagnosis is intended to assist by generating additional realistic image visualization and a better distinction among different tissues.
\end{abstract}

Keywords: radiance, silhouette mode, lipoleiomyoma, 3D ultrasound

\section{Introduction}

Uterine lipoleiomyoma is an uncommon benign tumor that is comprised of diverse mixtures of smooth muscle and mature fat tissue [1]. They are characteristically found in women ranging from 50 to 70 years of age and they are accompanying with conventional leiomyomas [1]. Clinical symptoms are similar to those if fibroid tumors of uterus [1]. The sonographic aspect of lipoleiomyoma is that of a heterogeneous hyperechoic mass somewhat surrounded by a hypoechoic rind. This rind is believed to represent a layer of myometrium surrounding the fatty component [1]. The diagnosis can be confirmed on MRI, which can specifically depict fat content within the tumor [2]. Here, we report a case of uterine lipoleiomyoma in a 49-year-old woman where three-dimensional radiance mode was applied to enhance its diagnosis. This is the first report in which this new ultrasonographic mode has been used to diagnose a gynecologic neoplasm.

Received 24.08.2015 Accepted 17.09.2015

Med Ultrason

2016, Vol. 18, No 2, 247-249

Corresponding author: Prof. Francisco Raga

Departamento de Obstetricia y Ginecología, acultad de Medicina y Odontología,

Universidad de Valencia

Avd. Blasco Ibañez no 15, 46010, Valencia, Spain Phone: 963864170 , Fax: 963864815

E-mail: francisco.raga@uv.es.

\section{Case report}

A 49-year-old African American female patient, gravida 4 , para 3, attended our gynecology outpatient department with complaints of lower abdominal pelvic pain, dyspareunia, and several months of heavy menstrual bleeding. There was no previous illness or surgical intervention. The patient was a non smoker with normal bowel habits. She was referred for an ultrasound (US) examination. This demonstrated an enlarged uterus with an anterior intramural hyperechoic mass measuring $5.2 \mathrm{~cm}$ in the largest diameter. Furthermore, two intramural leiomyomas measuring 5 and $4 \mathrm{~cm}$ in the largest diameter were also detected. Three-dimensional (3D) ultrasound Radiance System Architecture mode (Voluson E10, GE Healthcare, Madrid, Spain) displayed a well-defined mass on the anterior wall of the uterus. Additionally, the $3 \mathrm{D}$ radiance mode depicted a fat component as a central translucent appearance mass clearly delimited by an intense peripheral capsule. On the basis of these findings, a uterine lipoleiomyoma was considered. MRI was performed, which confirmed the 3D-ultrasound findings. The patient underwent a hysterectomy, and the histopathological examination confirmed the diagnosis of a uterine lipoleiomyoma (fig 1).

\section{Discussions}

Great effort has been made in the recent past to develop new diagnostic tools. Consequently, the last decade 


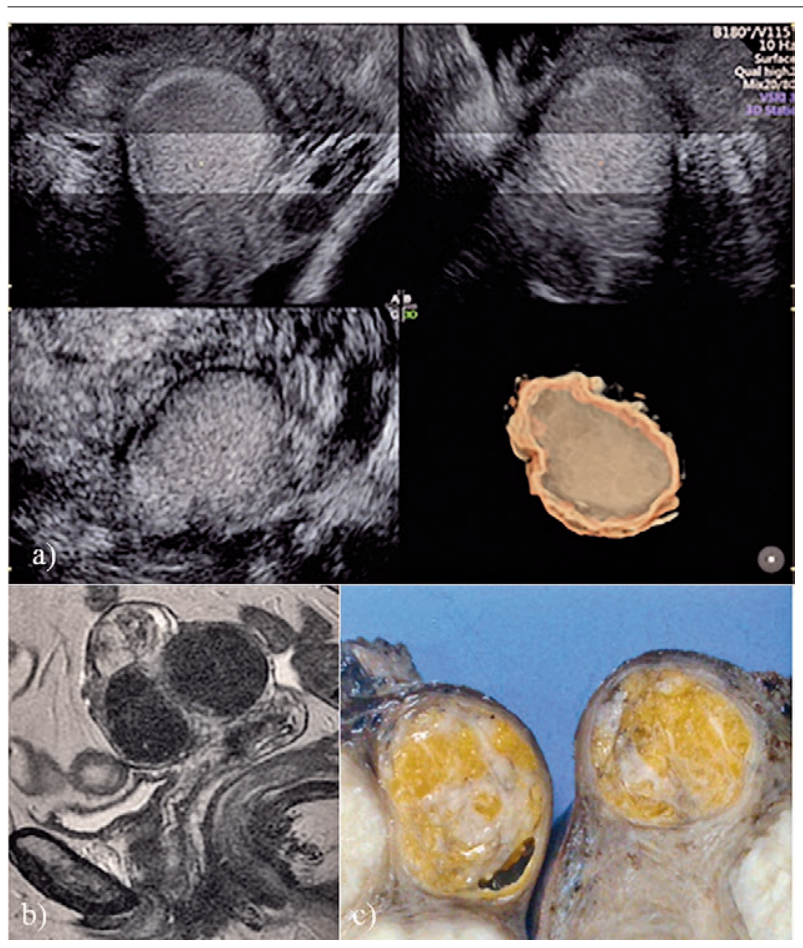

Fig 1. a) Three-dimensional radiance mode rendered image revealing a lipoleiomyoma. The peripheral rind represents a layer of myometrium surrounding the central fatty component; b) MRI shows a homogeneous, hyperintense intramural mass with a well-defined margin and a peripheral low signal intense rim corresponding to a layer of myometrium surrounding the mass; c) Histopathological examination confirming the diagnosis of a lipoleiomyoma.

has witnessed an unprecedented growth of different ultrasound imaging modalities [3]. Recently, radiance has been incorporated to ultrasound equipment (Voluson ${ }^{\mathrm{TM}}$ E10, GE Medical Systems) as a new imaging render mode.

Radiance development started in 1984 at Lawrence Berkeley National Laboratory (Berkeley, CA) [4]. Radiance has been used by architects and engineers to predict illumination, visual quality and appearance of innovative designs, as well as by researchto evaluate new lighting technologies [5-7]. It is based on a backward ray tracing algorithm [5]. This means that light rays are traced in the opposite direction to that which they naturally follow. Therefore, the process stars from a viewpoint and then traces the rays up to the light sources taking into account all physical interactions (reflection, refraction, etc) with the surfaces of the objects compositing the images [5-7]. Additionally, radiance implements global illumination using the Monte Carlo method to sample light falling on a point [5-7]. Monte Carlo methods are a category of computational systems that involve the random sampling of a quantity [5-7]. This method has become popular for simulating light transport in tissues for more than three decades [5], because it provides a flexible and rigorous solution to the problem of light transport in complex structures. Therefore, nowadays this method is viewed as the gold standard method to model light transport in tissues [5-7].

The incorporation of 3D-ultrasound to prenatal diagnosis, gynecology and reproductive medicine, has been a real revolution in these fields of medicine [8-11]. The evolution of this ultrasonographic modality has been remarkable in recent years. In addition, the recent advent of 3D-ultrasound HDlive (High Definition) technology has been an outstanding improvement in the quality of the 3D-images obtained [8-10]. HDlive uses an adjustable light source and software that calculates the propagation of light through surface structures in relation to the light direction [8-11]. Moreover, HDlive enhances the depth perception throughout the development of lighting and shadowing effects [8-11].

The introduction of the radiance or silhouette mode as a new tool in ultrasound diagnosis represents again a new breakthrough, as it is intended to aid in ultrasound diagnosis by creating a more realistic visualization and a greater contrast between different tissues. Recently, the prenatal diagnosis of a circumvallate placenta has been reported using this innovative ultrasonographic mode [12]. We now are beginning a fascinating journey in order to find the different applications of this new technology not only for prenatal diagnosis but also for obstetrics, gynecology and reproductive medicine. Certainly, radiance ultrasound technology offers many applications in these fields of medicine in the future and our case is a clear example of this breaking ground technology.

\section{References}

1. Aung T, Goto M, Nomoto M, et al. Uterine lipoleiomyoma: a histopathological review of 17 cases. Pathol Int 2004; 54: 751-758.

2. Tyagi N, Tyagi R, Griffin Y. Uterine lipoleiomyoma. BMJ Case Rep 2014 Dec 4. doi: 10.1136/bcr-2014-207763.

3. Bonilla-Musoles F, Raga F, Osborne NG, et al. Multimodality 3-dimensional volumetric ultrasound in obstetrics and gynecology with an emphasis in HDlive technique. Ultrasound Q 2013; 29: 189-201.

4. Ward GJ, Rubinstein FM. A new technique for computer simulation of illuminated spaces. LEUKOS 1988; 17: 8090.

5. Zhu C, Liu Q. Review of Monte Carlo modeling of light transport in tissues. J Biomed Opt 2013; 18: 50902.

6. Hayashi T, Kashio Y, Okada E. Hybrid Monte Carlo-diffusion method for light propagation in tissue with a lowscattering region. Appl Opt 2003; 42: 2888-2896. 
7. Wu L, Zhang G, Luo Q, Liu Q. An image-based rat model for Monte Carlo organ dose calculations. Med Phys 2008; 35: 3759-3764.

8. Grigore M, Mareş A. The role of HDlive technology in improving the quality of obstetrical images. Med Ultrason 2013; 15: 209-214.

9. Raga F, Castillo JC, Bonilla F Jr, Bonilla-Musoles F. HDlive ultrasound images in assisted reproduction treatment. Reprod Biomed Online 2013; 26: 269-271.
10. Raga F, Llinares C, Cholvi S, Bonilla F, Pascual C, Cano A. HDlive imaging of cystic uterine leiomyma degeneration. Ultrasound Obstet Gynecol 2015 Aug 19. doi: 10.1002/ uog. 15727.

11. Grigore M. HDlive imaging of a serous bortherline ovarian tumor. Ultrasound Obstet Gynecol 2013; 41: 598-599.

12. AboEllail MA, Kanenishi K, Mori N, Kurobe A, Hata T. HDlive image of circumvallate placenta. Ultrasound Obstet Gynecol 2015; 46: 513-516. 